\title{
Analysis of Scaling Behaviour of ECG Signal during Atrial Fibrillation
}

\author{
LT Mainardi $^{1}$, R Sassi $^{2}$ \\ ${ }^{1}$ Dipartimento di Bioingegneria, Politecnico di Milano, Milano, Italy \\ ${ }^{2}$ Dipartimento di Tecnologie dell'Informazione, Università di Milano, Crema, Italy
}

\begin{abstract}
In this study, surface ECG signals recoded during Atrial Fibrillation $(A F)$ episodes have been investigated to assess the presence of scaling behaviors. The ECG signals consisted of one minutes recordings coming from the 2004 Computers in Cardiology Challenge database. After having removed the ventricular related activity, the residual ECG signal (rECG) was analyzed using Detrended Fluctuation Analysis (DFA). We documented the existence of two scaling regions in the signals. Over long time scales $(n \geq 32, \approx 234 m s)$ the $r E C G$ fluctuations resembled a white or slightly persistent noise (scaling $A=0.64 \pm 0.17)$, whereas over short scales $(n \leq 16, \approx 125 \mathrm{~ms})$ slowly decaying antipersistent correlations were evident (scaling $A=1.20 \pm 0.20$ ).

In addition, we searched for possible differences in the scaling exponents between terminating $(T)$ and nonterminating (N) AF episodes. We observed that both short-term (Al: $1.29 \pm 0.15$ for $T$ vs. $1.12 \pm 0.18$ for $N$, $p<0.005)$ and long-term $(A 2: 0.54 \pm 0.16$ vs. $0.70 \pm 0.14$, $p<0.005)$ scaling exponents were significantly different $(t$-test).
\end{abstract}

\section{Introduction}

Lately, several experimental results suggested that Atrial Fibrillation (AF) might not be a purely random phenomenon. The evidences of patterns of spatial and temporal organization have been documented in endocardial/epicardial atrial recordings using linear and non-linear signal processing techniques. Shortly, linear correlation was found to decrease exponentially with the distance between recording sites [1]; entropy and various regularization indexes uncovered a short-term temporal organization of the atrium and its changes with different type of AF episodes (Wells classes) or drugs' infusions [2-3]. In addition, Hoekstra [4] observed scaling regions and measured the correlation dimension of epicardial atrial recording during AF. All these findings suggest the existence of organized patterns of electrical activity in the atria during AF. The level and the extent of the organized activity might be related to the number of circling wave fronts which travel in the atria and probably to the selfsustainability of AF episodes [5].

In the analysis of surface ECG signals, the research has been traditionally focused on the quantification of oscillatory components in the residual ECG signal (rECG, i.e. an ECG signal where ventricular activities had been, removed through beat averaging techniques [6-7]). But to the extent of our knowledge, no systematic attempt has been made to search for atrial organized activation patterns manifesting in scaling behaviour and long-term correlations properties of the rECG signal.

Scaling properties have already been observed in a variety of biological signals and data [8-9] and various methodological approaches have been pursued. Among them: Detrended Fluctuation Analysis (DFA) [10], Wavelet-based [11] and spectral estimators [12]. DFA is likely to be the most used method as its implementation is straightforward. It is robust and blind to superimposed linear trends [10] (a property shared with most wavelet based tools, which, though often more accurate, are unfortunately more prone to computational and methodological pitfalls).

In this work, we analyze rECG signals using DFA in search of scaling behaviours during $\mathrm{AF}$ episodes. We examined AF episodes (1 minute long) coming from the PhysioNet AF Dataset (Computers in Cardiology Challenge 2004) [16]. Additionally, possible differences between scaling properties of terminating $(\mathrm{T})$ and nonterminating $(\mathrm{N})$ episodes of AF were investigated.

\section{Methods}

Database

We analyzed data coming from the 2004 Computers in Cardiology AF Termination Challenge Database [16]. The Database includes 80 1-minute records, extracted from long-term ECG Holter recordings of patients undergoing Atrial Fibrillation events. Each recording was sampled at $128 \mathrm{~Hz}$ and two simultaneous leads were made available. The database is divided in different subsets. A more detailed description can be found in [13]. We used data for terminating and non-terminating $\mathrm{AF}$ 
events. Briefly, the database's authors labelled "nonterminating" $(\mathrm{N})$, those records for which AF lasted for at least one hour after the end of the record, and "terminating" (T) records for which AF terminated within one second after the end of the record. In details, we analyzed 50 records: the whole test set $\mathrm{A}$ (30 records, a01 to a30) and 20 records contained into the training-set (records n01 to n10 and t01 to t10).

\section{Processing of ECG signals}

Extraction of the residual ECG was obtained through beat-to-beat subtraction of an averaged QRST complex [6-7]. The algorithm we actually employed was a refined version of the one used for the 2004 competition [19].

Despite the 2004 Challenge dataset already contained QRS annotations, ventricular beats were mainly misclassified, leading to a serious source of cancellation errors. Thus, a slightly modified version of OSEA, a freely available ECG library [14], was employed to detect and classify beats. QRS onsets and widths were further refined by means of the publicly available software, ECGPUWAVE [15]. Then, on a lead-by-lead basis, separate average templates were built for QRS and T waves. To take into account morphological changes and minor variations in the electrical axis induced by respiration, subtraction of the templates was performed after a warping procedure and consecutive templates were connected via linear interpolation. With the assumption that during fibrillation atrial and ventricular activities are highly independent, the resulting rECG includes atrial activity only. The quality of the subtraction procedures was monitored computing the average cross-correlations among the templates and the ECGs (quality index, $Q A$ ).

\section{Detrended fluctuation analysis}

Detrended Fluctuation Analysis (DFA) is a method widely employed to detect long-range correlations embedded in seemingly non-stationary time series. It emplaces a robust approach which avoids most spurious detections of apparent scaling behaviours (especially the ones arising with slowly varying trends) [10].

In details, the original signal $x(k)$ is firstly integrated

$$
y(k)=\sum_{i=1}^{k}\{x(i)-E[x]\}
$$

where $E[x]$ is the signal average (see figure 1a). Next, the integrated time series is divided into boxes of equal length $n$. In each $\mathrm{i}^{\text {th }}$ box, the local trend $y_{i, n}(k)$ is evaluated by linear least-square fitting (figure $1 \mathrm{~b}$ ) and then $y(k)$ is detrended by subtracting $y_{i, n}(k)$. Finally, the root meansquare fluctuation of the resulting detrended time series is calculated by

$$
F(n)=\sqrt{E\left[\left(y(k)-y_{i, n}(k)\right)^{2}\right]}
$$

This computation is repeated over all time scales (box size $n$ ) to provide a relationship between $F(n)$, the average fluctuation, and the box size $n$. If this relationship is linear on a log-log plot, it then indicates the presence of power law scaling, that is

$$
F(n) \approx n^{A} \Rightarrow \log F(n) \approx A \log n
$$

where $A$ is the scaling exponent. $A$ is computed by linear regression on a log-log plot.

For fractional Gaussian noise (fGn), which is a stationary signal, $A$ corresponds to the Hurst exponent, as obtained, e.g., with the rescaled range R/S analysis [17]. The match among the two indexes does not hold instead for fractional Brownian motion, which is a non-stationary signal obtained integrating fGn. For this second class of well known signals, $A=H+1$. In some cases, more than
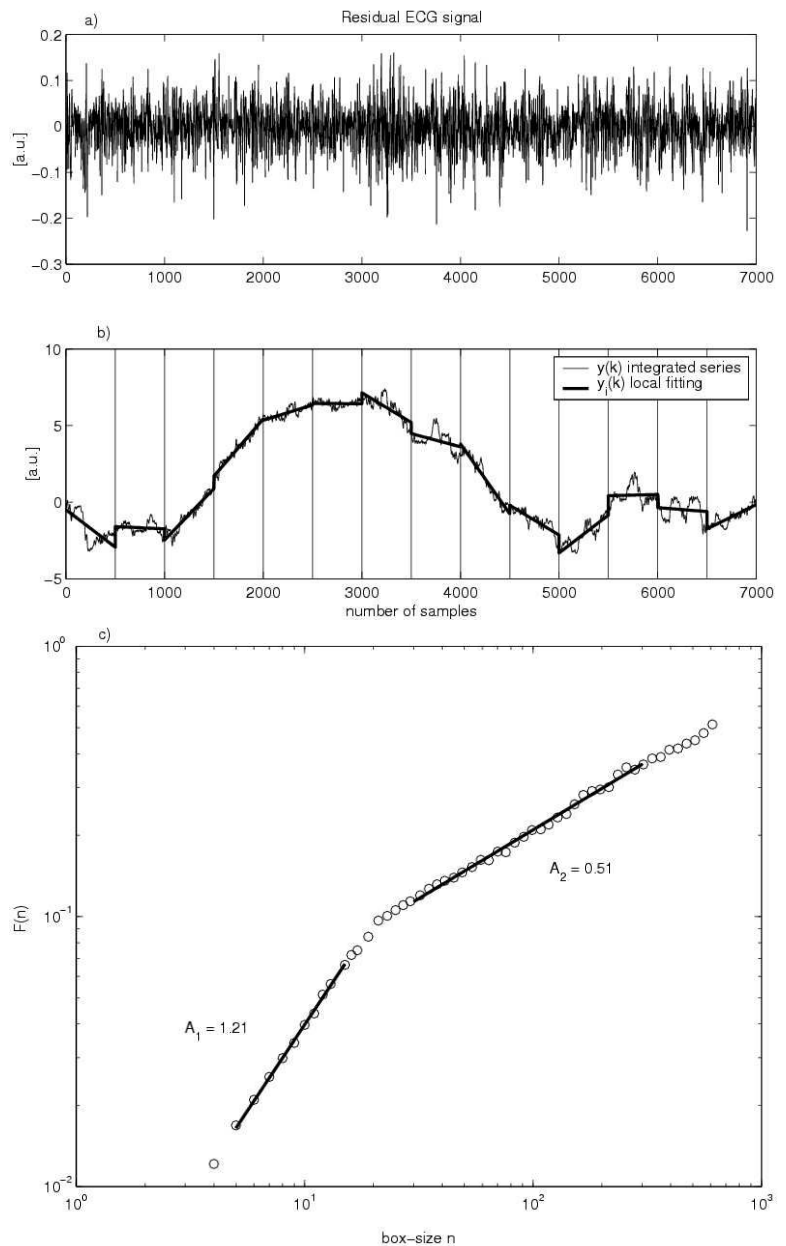

Figure 1. Application of DFA analysis to residual ECG signal (panel $a$ ). The signal is integrated (panel $b$ ) and divided in boxes of equal size ( $n=500$ in the plot). The local trend (bold line) is then removed in each block and $F(n)$ is computed (see text for details). The procedure is repeated changing the box-size to build the curve in (panel $c$ ). The values of the scaling exponents are computed by linear regression. 
one scaling region might be detected in the relationships among $F(n)$ and $n$.

\section{Results}

All the records comprised in the database were suitable for residuals ECG series construction and DFA analysis. Of the two leads available for each record, DFA was computed on the one where the rECG construction had been more effective (higher quality index, $Q A$ ).

DFA was computed for scales $n$ which ranged between 4 and 600 , using the algorithm freely available from Physionet. Figure 2 shows a comprehensive view of the average fluctuations.

The relationship among $F(n)$ and $n$ evidenced a crossover phenomenon between a region (short scales, $4 \leq n \leq 16$ ) where the scaling exponent $A_{l}$ was larger and a second region (large scales, $32 \leq n \leq 256$ ) with a smaller scaling exponent $A_{2}$. The crossover was present in nearly all the records as evinced from figure 2, where most of the curves lie in the central darker strip.

The values of $A_{l}$, though not strictly constant among different recordings, varied in a limited range. The average values of the two scaling parameters computed over the entire population were: $A_{l}=1.20 \pm 0.20$ and $A_{2}=0.64 \pm 0.17$. They suggest that for long time scales, the rECG was quite random $\left(H \equiv A_{2}\right)$, resembling a white noise (for which $H=0.5)$ or slightly persistent noise $(H>0.5)$. On the other side, at smaller scale, rECG was similar to an antipersistent fractional Brownian motion $\left(H \equiv A_{l^{-}}\right.$ $1=0.20 \pm 0.20$ ), with correlations which decay slowly. Signals with slowly decaying correlations are often

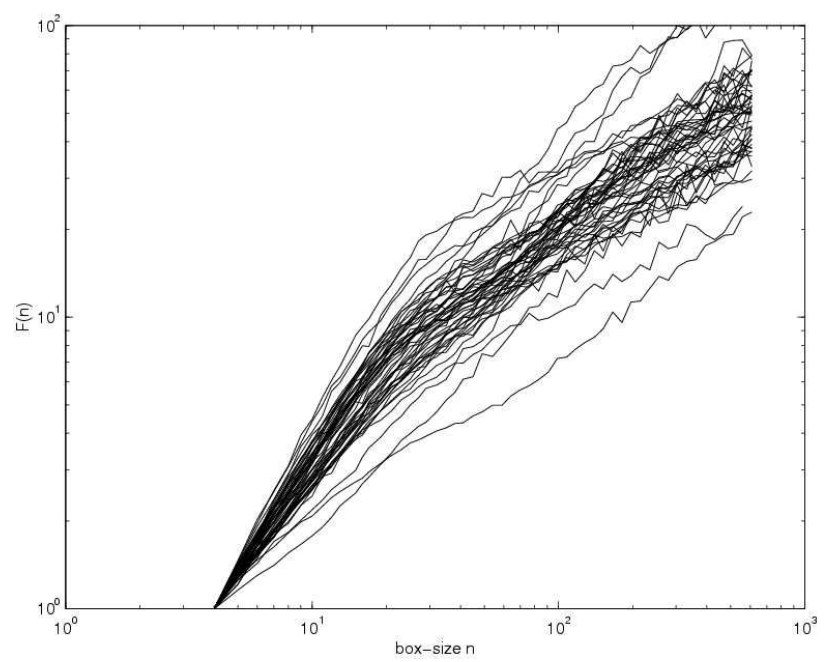

Figure 2. Results of DFA analysis for all the recordings contained in the dataset. The presence of two scaling regions is evident in almost all the records. Results were normalized to fit them in a single graph.

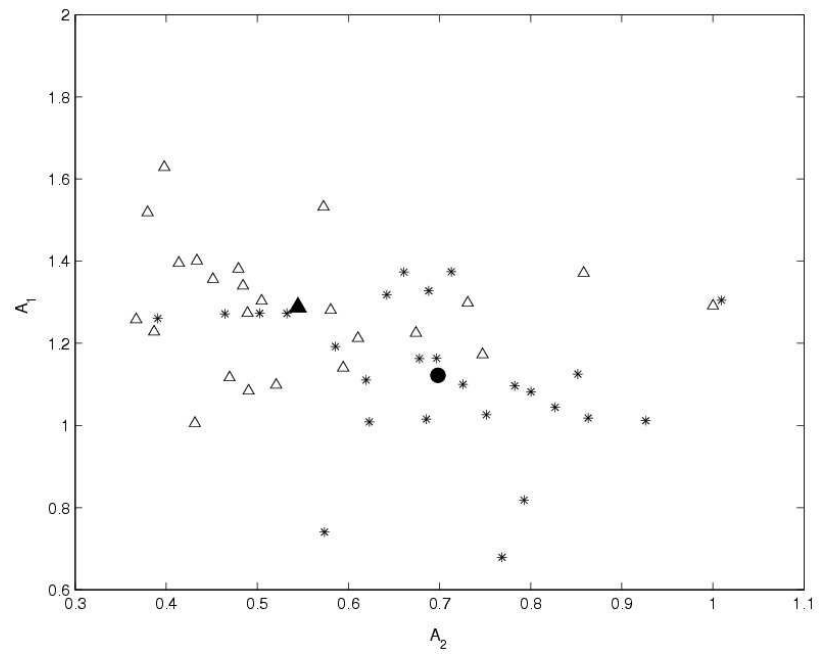

Figure 3. Scatterplot of the scaling exponents in terminating (triangles) and non-terminating (stars) AF episodes. Each symbol represents one record and the location on the graph is determined by the two scaling exponents A1 and A2. The dark triangle and circle mark the average values of each group (the triangle is for terminating AF episodes).

referred to as " $1 / f$ noise".

The location of the crossover among the two different regimes (i.e. the point were the curves changed slope), might be used to obtain a rough estimate of the temporal extent of the correlations in the rECG signal. In figure 2 the crossover is located approximately between $n=20$ and $n=30$, which corresponds to a window length of $160-240$ ms.

A second step in the work was the comparison among terminating and non-terminating AF episodes. Interesting differences in the scaling behaviour were observed; the results are presented in Figure 3. In particular, we observed that the exponent $A_{l}$ was larger during terminating episodes $\left(A_{l}=1.29 \pm 0.15\right.$ for $\mathrm{T}$ vs $1.12 \pm 0.18$ for $\mathrm{N}$, t-test $p<0.005)$. On the contrary, $A_{2}$ was smaller $\left(A_{2}=0.54 \pm 0.16\right.$ for $\mathrm{T}$ vs $0.70 \pm 0.14$ for $\mathrm{N}$, t-test $\left.p<0.005\right)$.

The populations in figure 3 form two quite distinct clouds, with the terminating episodes preferring the upper-left corner. Even if the separation among the two populations was not complete in the $A_{I}-A_{2}$ space, and overlaps exist between the two groups, the analysis of the scaling exponents of rECG seems to be a promising tool for a correct classification of each $\mathrm{AF}$ episode.

\section{Discussion and conclusions}

We investigated the scaling behaviours of rECG signals obtained from patients undergoing Atrial Fibrillation episodes. The analysis, performed with DFA, evidenced the existence of two scaling regions and a 
crossover phenomenon. The signal resembled a white noise process for long scale $(n>30)$. When smaller scales were considered $(n<20)$ slowly decaying correlations appeared and the rECG was similar to an anti-persistent fractional Brownian motion. These findings could be interpreted taking into account that, during $\mathrm{AF}$, multiple wave fronts propagate continuously through the right and left atria, circling around anatomical and functional barriers [18]. Over short time scales, we might suppose that each point in the atria is crossed by the same wave, and that the electrical activity is locally organized. It is therefore likely for the resulting surface rECG signal not to be completely uncorrelated. However, when the observation time is extended, new fibrillatory waves may be randomly generated while others die out. Now a single local area of the atria experiences the crossing of several different wave fronts and the resulting temporal activation is then more complex and erratic. It would not be surprising for the surface ECG to resemble, in this case, a white noise process. We may also speculate that the scale at which the transition between a surface rECG signals with slowly decaying correlations and a white noise appears, could be an indirect indication of the fibrillation waves mean life-time.

The observed scaling phenomenon was found in nearly all the records we analysed. In addition, the scaling properties were different in $\mathrm{N}$ and $\mathrm{T}$ episodes, suggesting that such kind of analysis could be capable of correctly distinguishing terminating and non-terminating $\mathrm{AF}$ episodes.

To the extent of our knowledge, the present paper is the first evidence of this phenomenon in ECG signals during AF. However, further studies are needed to provide a physiological interpretation of these findings.

\section{References}

[1] Botteron GW, Smith JM. A technique for measurement of the extent of spatial organization of atrial activation during atrial fibrillation in the intact human heart. IEEE Trans Biomed Eng 1995;42:579-586.

[2] Mainardi L, Porta A, Calcagnini G, Bartolini P, Michelucci A, Cerutti S. Linear and non-linear analysis of atrial signals and local activation period series during atrial-fibrillation episodes. Med Biol Eng Comput. 2001 Mar;39:249-54.

[3] Mainardi L, Corino VDA, Lombardi L, Tondo C, Mantica M, Lombardi F, Cerutti S,.Assessment of the dynamics of atrial signals and local atrial period series during atrial fibrillation: effects of isoproterenol administration, Biomedical Eng. OnLine, 2004, 3:37.

[4] Hoekstra BPT, Diks CGH, Allessie MA, De Goede J. Nonlinear analysis of epicardial atrial electrograms of electrically induced atrial fibrillation in man. J Cardiovasc Electrophysiol 1995;6:419-440.

[5] Shan Z, Van Der Voort PH, Blaauw Y, Duytschaever M, Allessie .MA. Fractionation of electrograms and linking of activation during pharmacologic cardioversion of persistent atrial fibrillation in the goat, (2004) Journal of Cardiovascular Electrophysiology., vol. 15, no. 5, pp. 572580 .

[6] Slocum J, Sahakian A, Swiryn S. Diagnosis of Atrial Fibrillation From Surface Electrocardiograms Based on Computer-detected Atrial Activity. J Electrocardiol 1992;25(1):1-8.

[7] Holm $M$, Pehrson $S$, Ingemansson $M$, Sornmo $L$, Johansson R, Sandhall L, Sunemark M, Smideberg B, Olsson C, Olsson SB. Non-invasive assessment of the atrial cycle length during atrial fibrillation in man: introducing, validating and illustrating a new ECG method. Cardiovascular Research 1998;38:69-81.

[8] Audit B, Thermes C, Vaillant C, d'Aubenton-Carafa Y,Muzy JF, Arneodo A. Long-range correlations in genomic DNA: A signature of nucleosomal structure, Phys. Rev. Lett. (2001), 2471-2474.

[9] Goldberger AL, Amaral LA, Hausdorff JM, Ivanov PCH, Peng CK, Stanley HE. Fractal dynamics in physiology: alterations with disease and aging. Proc Natl Acad Sci U S A. 2002 Suppl 1:2466-72.

[10] Peng C-K, Havlin S, Stanley HE, Goldberger AL. Quantification of scaling exponents and crossover phenomena in nonstationary heartbeat time series. Chaos 1995, 5:82-87.

[11] Audit B, Bacry E; Muzy JF; Arneodo A. Wavelet-based estimators of scaling behavior Information Theory, IEEE Transactions on 2002, 11:4, 2938 - 2954

[12] Geweke J, Porter-Hudak S. The estimation and application of long-memory time series models. J. Time Ser. Anal., 1983, 4: 221-238

[13] Moody GB. Spontaneous Termination of Atrial Fibrillation: A Challenge from PhysioNet and Computers in Cardiology 2004 Computers in Cardiology, 2004 31:101-104.

[14] Pat Hamilton, Open Source ECG Analysis Software, EP Limited, freely available from www.eplimited.com

[15] Laguna P, Jané R, Bogatell E, Anglada DV. ECGPUWAVE, freely available from www.physionet.org.

[16] AF Termination Challenge database, 2004 Computers in Cardiology, www.physionet.org/challenge/2004.

[17] Mandelbrot BB, The fractal geometry of nature, Freeman, New York.

[18] Allessie M, Lammers WJEP, Bonke FIM, Experimental evaluation of Moe's multiple wavelets hypothesis of atrial fibrillation. In Zipes DP, Jalife J. eds: Cardiac Electrophysiology and Arrhythmias. 1985, Grune and Stratton Inc., New York. pp.265-275.

[19] L.T. Mainardi, M. Matteucci, R. Sassi, On Predicting The Spontaneous Termination Of Atrial Fibrillation Episodes Using Linear And Non-Linear Parameters Of ECG Signal And RR Series, Computers in Cardiology 2004, 31:665668

Address for correspondence:

Ing. Luca T. Mainardi, PhD

Department of Biomedical Engineering

Polytechnic University

Via Golgi 39, 20133 Milano, Italy

E-mail: luca.mainardi@biomed.polimi.it 